\title{
ТЕХНОЛОГИЯ МАСТЕРСТВА
}

УДК $7 / 78$

doi: $10.17223 / 26188929 / 10 / 9$

\section{Галина Схаплок (Кайя Саариахо)}

\section{ФОРМИРОВАНИЕ КОМПОЗИЦИОННОЙ СЕТИ С ПОМОЩЬЮ КОМПЬЮТЕРА}

\begin{abstract}
Данная статья представляет собой перевод музыковедческого эссе выдающегося композитора современности Кайи Саариахо, в котором она рассказывает о возможностях специально созданной компьютерной программы для работы со звуковым материалом. Автором освещается специфика творческого процесса, связанная с созданием многоуровневой композиционной сети, использованием техники интерполяции, моделированием звуковых объектов и исследованиями в области тембра. Для широкого круга любителей музыки и профессионалов.
\end{abstract}

Ключевые слова: CHANT, звук, параметры, тембр, интерполяция.

Мне хотелось бы сосредоточиться в данной статье на описании моего музыкального мышления, касающегося сочинения музыки с использованием компьютера, и рассказать, опираясь на конкретные примеры, о внедрении ходовых принципов работы в программе, созданной мною для композиторской практики.

Программа была реализована с помощью системы FORMES, разработанной Ксавье Роде и Пьером Конте, применяемой на компьютере VAX 11/780 в институте IRCAM, и имеет возможность соединения со звуковым синтезом и обработкой данных в программе CHANT на матричном процессоре FPS-100. Все звуковые примеры синтезировались в той версии программы CHANT, которая была создана Ивом Потаром и Яном Вандехидом. Данная версия включала волновую формантную функцию синтезированной техники (далее именуемой FOF), разработанную Ксавье Роде (см. журнал «Компьютерная музыка» 1984, выпуск 8/3), а также банк изменяющихся во времени фильтров. Управление фильтрами осу- 
ществлялось так же, как и формантами, контролируемыми обычно в программе CHANT, т.е. путем указания центральной частоты, полосы частот и амплитуд. Это дало возможность использования программы CHANT не только для синтеза, но и для фильтрации и обработки звука. Можно было бы использовать любой внешний источник, но в этой работе я применяла фильтры только с шумовым источником звука.

Хотелось бы выразить особую благодарность Ксавье Роде и Жан-Батисту Баррьеру за помощь в реализации моей программы; она помимо музыкальных разработок потребовала и множество новых решений с точки зрения программирования.

Данная работа началась с решения вопросов, связанных с организацией звукового материала. Мне хотелось понять, может ли композитор оперировать музыкальными элементами, не заблудившись в бесконечной сети алгоритмов. Производство интересного звука предъявляет высокие требования к нему [композитору], а сама композиционная работа с синтетическим (искусственным) материалом часто остается второстепенным делом.

Я сформировала на свой вкус некоторые первичные модели для музыкальных ситуаций. Суть моделей заключалась в возможности управления различными музыкальными параметрами с учетом их особенностей. Интересно было узнать, насколько далеко может завести рассмотрение разнообразных параметров начиная с одной и той же точки отсчета. Также хотелось иметь возможность реализовать две ситуации различного типа в одно и то же время:

- постепенную интерполяцию между заданными точками;

- резкие переходы между различными музыкальными характеристиками.

Долгое время меня интересовала идея музыкальной интерполяции наряду с другими процессами. Моя начальная точка в профессиональном развитии частично определялась данными интересами по отношению к различным конкретным музыкальным параметрам.

В основном мои программы состоят из моделей (паттернов), которые существуют в качестве списка значений для выбранного параметра и в дальнейшем отображаются вместе. Ниже будут перечислены эти списки и комплекты матричных списков. Кроме них могут использоваться различные функции времени для определения общих эволюционных процессов и контуров. В матрице опре- 
делены интерполяционные отношения и значения приведены к желаемому числу частиц:

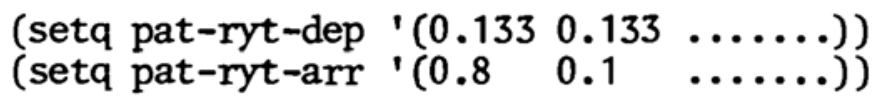

Рис. 1

В рамках одного звука простая интерполяция может быть осуществлена между двумя значениями $(0.133 \rightarrow 0.8)$ или между кольцевой матрицей, где каждое из значений, когда оно повторяется, изменяется, что ведет к непрерывной трансформации общего характера модели:

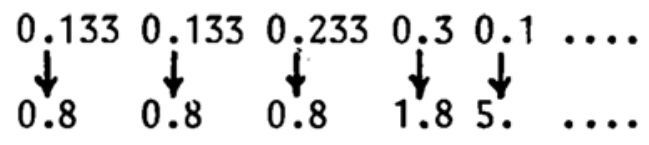

Рис. 2

Такого рода циркулярный список может быть, например, у аккордов из паттернов, где каждый звук в процессе совершает глиссандо к звуку нового аккорда.

Моей целью является создание многоуровневой сети постоянно изменяющихся, но подконтрольных элементов. С помощью этих средств я могу также управлять в различных объемах теми же параметрами как изнутри, так и снаружи звука.

Обычно берется постоянно меняющийся, но целенаправленный процесс, полученный путем объединения различных матриц всевозможных параметров:

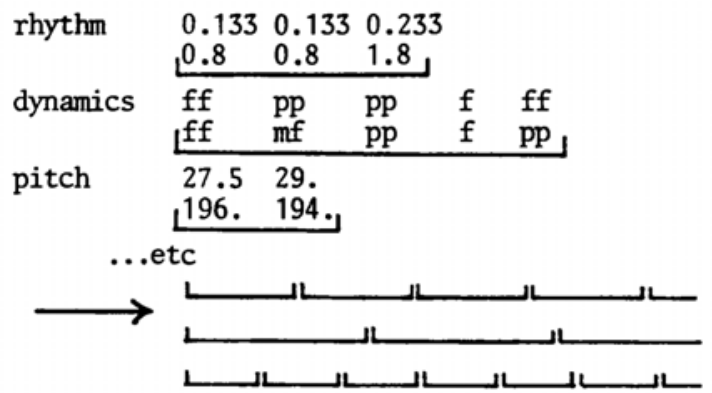

Рис. 3 
Легче управлять более крупными единицами, не теряя представление о ситуации на микроуровне. Это дает возможность осуществлять контроль над музыкальными формами и концентрировать внимание на разнообразных принципах формообразования.

Теперь хотелось бы представить ритмическую часть этой программы, которая как раз таки адаптирована в качестве основы для обработки других различных параметров. Смысл заключается в создании определенных условий, при которых можно повторить тот же ритмический паттерн с плавным изменением темпа и на разной скорости с целью осуществить интерполяции между группами различной протяженности и, возможно, различным количеством частиц. Мною владело желание реализовать свои музыкальные фантазии, которые не составляют сложности для компьютера и весьма для него характерны, но невозможны как таковые для осуществления в инструментальной музыке. Другой интересный аспект, связанный с этим, - это исследование компьютерной и исполнительской интерпретации, к примеру, тех же ритмических процессов в этих двух областях, из-за их различной природы ставящей акцент на различных аспектах данной модели. Впервые воплотить такие идеи мне удалось в сочинении «Verblendungen» (1982-1984) для оркестра и компьютерной ленты, и я предвижу много дополнительных возможностей в этом направлении.

В процессе работы я должна была в первую очередь модифицировать установку длительностей ритмических паттернов и ритмической матрицы, в которой определены отношения между различными частицами:

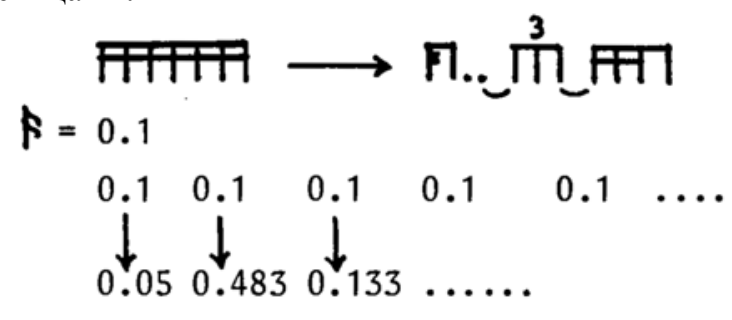

Рис. 4

Данный рисунок - пример ритмического процесса, в котором быстрое остинато понемногу изменяет свой профиль. Скорость интерполяций в пределах общей продолжительности может регули- 
роваться заданной функцией времени, а различные фазы преобразования могут быть усилены, придавая им различную продолжительность.

Следующие ритмические интерполяции значительно отличаются от предыдущих особой органичностью, метафоричностью процесса. Эта интерполяция происходит между двумя группами, которые имеют разное количество частиц. Данный процесс более капризный, чем описанный выше, ему труднее придать форму. Ниже представлен простой пример ровного остинато из пяти нот, переходящего в остинато из семи нот таким путем, что две ноты просто разделены на два значения:
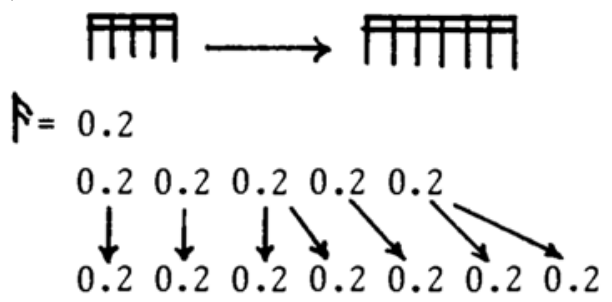

Рис. 5

Исходя из этой первичной ритмической модели, программа применима и к другим музыкальным параметрам. Отныне все параметры, к которым относятся такого рода процессы, могут включаться в установленные лимиты этой программы.

В дальнейшем мне бы хотелось адаптировать это и к контролю динамики. Динамика различной продолжительности как независимая группа (нежели, к примеру, ритм) могла бы сформировать постоянно меняющиеся акценты на ритме.

Что касается тембра, можно выбрать некоторые параметры для матричной группы, а другими управлять с обычными временными функциями и барьерами в зависимости от необходимости и нюансов. Одна из моих задач в отношении тембра - разработка точного тембрового изменения в каждом отдельном тоне. Такой вид пуантилистической фактуры может быть объединен с интерполяциями более широких линий, встречающихся в пределах других тембровых факторов или параметров. Я нашла одно применение этой идеи с тем, что я называю растяжение-матрица, где спектральные контуры изменяются посредством растягивания и сжатия формантных 
частот (см. примеры ниже). Здесь каждая частица паттерна имеет формантную структуру, которая отличается от предшествующей.

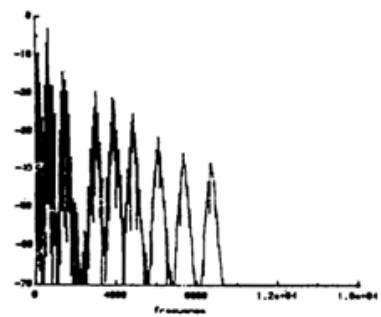

Рис. 6. Спектральный контур в своей первоначальной форме

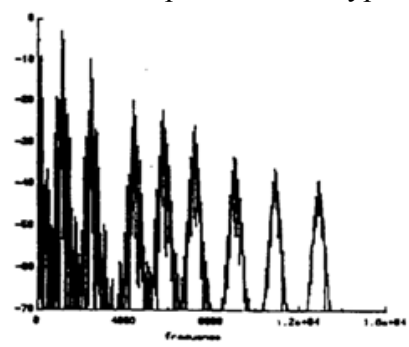

Рис. 7. Растянутая версия

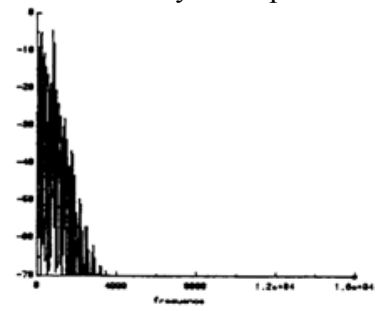

Рис. 8. Сжатая версия

B технике синтеза FOF, где формантная пропускная способность магнитной ленты сокращается и приближается к нулю, они становятся частичными. Это пример применения аддитивного синтеза с программой CHANT, а также интересный способ создать ингармоническое звучание, используя какую-либо спектральную огибающую и уменьшая формантную пропускную способность ленты. 
В своей работе я применяю различные типы иерархий для эволюционных процессов частотных полос; можно выстроить общую эволюцию в течение одной фразы или группы событий, а также внутри одной ноты.

Особенностью программы CHANT является легкость, с которой можно работать над преобразованиями физической модели голоса или какого-либо другого музыкального инструмента и получить абстрактные звуковые объекты, далекие на слух от оригинала. Для меня эта ось является одним из параметров в тембровой области. Я объединяю, например, дыхание и голос как материалы с очень яркими, колоколообразными красками. Время затухания звучности или резонанса является вариабельным и может длиться долго.

Матрица дает бесконечно много возможностей для управления тембром. В качестве примера я выбрала вариант работы только с одной спектральной огибающей, для которой использовала различные сжимающие паттерны программы FOF и фильтры.

Такой способ достижения звука, «открытие», сделанное независимыми параметрами, как и в случае с физической структурой, далек от традиционного музыкального материала. К примеру, я создала звуки, в которых различные тембровые элементы сосуществуют независимо, в то время как в рамках одного и того же звука сочетаются два различных времени. Примером этого является звучание, где растяжение-матрица контролирует форманты фильтра, адаптируясь к длительности, предписанной ритмической матрицей. Шум следует заданным контурным обозначениям без количественных изменений.

Такой вид работы напоминает оркестровку на микроскопическом уровне. Это весьма интересно, так как дает возможность мыслить свободно от некоторых оков. Идея реализации определенных форм, как в макро-, так и в микроструктуре вполне возможна в отношении тембра и гармонии. Речь идет о принятии решений, которые являются музыкально значимыми. Такого рода многомерные звучания вызывают трудности в вертикальной и горизонтальной организации. Движение внутри звуков и высотные отношения, доступные слуховому восприятию, действительно требуют отказа от традиционных моделей организации. Кажется неестественным подчинить подобный материал обычным схемам, равно как было бы странным вписать конкретную музыку в рамки традиционной 
теории музыки или, скажем, хорового искусства. С другой стороны, не верится, что есть причины отказываться от всех традиционных измерений в пользу новых. Скорее, надо подходить открыто, не теряя музыкального чутья, к новым решениям, постепенно разрастающимся благодаря новым носителям информации.

Для меня в настоящее время звуковысотная организация является одной из самых сложных тем в компьютерной музыкальной композиции просто потому, что эти возможности неисчерпаемы и весьма доступны, и необходим творческий подход, чтобы изучить их. В дальнейшем я продолжу поиски способов непрерывного управления внутренней и внешней структурой звука, где гармония легко могла бы ассимилироваться в тембре. Сила гармонического мышления заключается в его возможности управлять формами и создавать напряжение. Вот почему я пришла к идее оперирования различными настройками и выявления способов перемещения от одной настройки к другой. Один из таких способов - ритмическая интерполяция, описанная выше, где один звук постепенно переходит в близлежащий звук новой настройки. Мне хотелось бы с помощью различных технических ресурсов найти новые эмпирические заменители для модуляций и ключей, посредством которых музыка могла бы быть окрашена в определенный оттенок. В любом случае, моя цель заключается в полном объединении гармонического мышления с тембровой организацией в моих сочинениях.

\section{Использованные источники:}

1. Saariaho, K. Shaping a compositional network with computer // Proceedings of the International Computer Music Conference. ICMC, 1984. P. 163-165.

\section{Galina Skhaplok (Kaija Saariaho)}

SHAPING A COMPOSITIONAL NETWORK WITH COMPUTER

Musical almanac of Tomsk State University, 2020, no. 10, pp. 93-101. doi: $0.17223 / 26188929 / 10 / 9$

The program has been entirely realized with Xavier Rodet's and Pierre Cointe's FORMES system which runs on IRCAM's VAX 11/780 computer. The version of CHANT includes the Formant Wave Functions synthesis technique as well as a bank of time-varying filters. I shaped in my mind some primary models for musical situations. Starting from these models, I intended to control various musical parameters, without forgetting their particular features. I have been interested since a long time in the idea of musical interpolation and processes. Within one sound, a simple inter- 
polation can be made between two values, or between the circular matrix where each value, when repeated, has changed, and thus continually changes the total character of the pattern. My object is to produce a multilevel network of continually changing but controlled items. Another interesting aspect - the same rhythmic process, since these two instruments, because of their different natures, give emphasis to different aspects of a given model. For example an even ostinato of five notes changes into an ostinato of seven notes in such a way that two notes are simply divided into two.

I wanted to adapt it next to the control of dynamics. One of my objectives concerning timbre is to produce a precise timbre change on each tone. This kind of pointillistic texture can then be combined with interpolations of greater line occuring within other timbre factors or other parameters. I created sounds where various timbre elements advance independently when two different times are used within the same sound. For me, the pitch organization is at present one of the most complicated themes in computer music composition, simply because the possibilities are endless and a very open, creative attitude is needed to explore them.

Keywords: CHANT, sound, parameters, timbre, interpolation.

\section{The used sources}

1. Saariaho, $K$. Shaping a compositional network with computer. Proceedings of the International Computer Music Conference, ICMC, 1984. P. 163-165. 